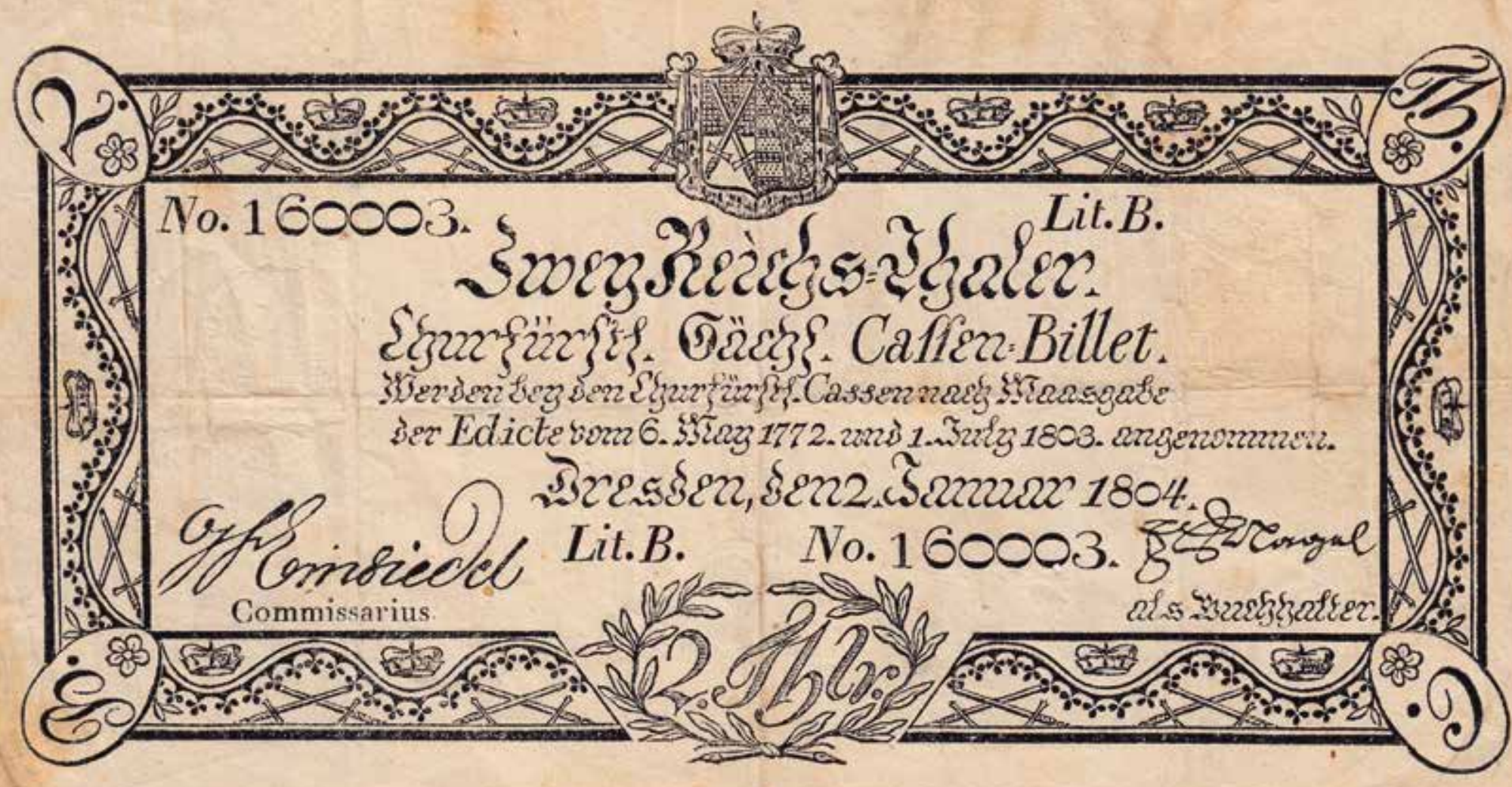

\title{
Papiergeld und Banknoten in Sachsen 1772 bis 1936
}

\author{
Frank Metasch
}

Kurfürstlich Sächsisches Kassenbillet, 2 Reichstaler, 1804 Foto: Frank Metasch
Wenn wir heutzutage etwas mit Bargeld bezahlen, so greifen wir ganz selbstverständlich auf die Banknoten und Münzen in unseren Portmonees zurück. Dass jedoch Banknoten als vollwertiges Geld zählen, ist erst eine jüngere Entwicklung vor allem des 20. Jahrhunderts. Bis dahin war im Verständnis der Zeitgenossen allein die „klingende Münze“, die ihren Wert in Form des enthaltenen Goldes und Silbers selbst in sich trug, wirkliches Geld. Viele Scheine trugen daher den Hinweis, dass sie jederzeit gegen „baares Geld“ eingetauscht werden konnten, so zum Beispiel bis in die 1870er Jahre hinein die Noten der Leipzig-Dresdner Eisenbahn-Compagnie. Selbst die Banknoten der deutschen Reichsbank waren bis 1909 keine gesetzlichen Zahlungsmittel, sondern ein Äquivalent der bei der Bank hinterlegten Goldmünzen, gegen die sie die Reichsbankhauptkasse in Berlin, dem aufgedruckten Zahlungsversprechen zufolge, jedem Einlieferer eintauschen würde.

Doch auch wenn Geldscheine noch im 19. Jahrhundert nicht als richtiges Geld galten, so waren sie im Zahlungsverkehr unerlässlich. Die zur Verfügung stehende Menge an Gold und Silber reichte schon lange nicht mehr aus, um den ständig steigenden Kapitalbedarf der sich rasant entwickelnden Wirtschaft oder auch der wachsenden Staats- haushalte abzudecken. Papierene Zahlungsmittel, mit ihren geringen Herstellungskosten, schienen vielen Staaten daher als ein ideales Mittel zur unbegrenzten Geldschöpfung. Gerade beim Papiergeld zeigte sich aber eine ganz besondere Zwiespältigkeit: Sinnvoll genutzt, bewährte es sich als ein wirkungsvolles Instrument bei der Sanierung der Staatshaushalte und bei der Überwindung von Finanz- und Kreditkrisen - im Übermaß eingesetzt, drohte hingegen die finanzielle und wirtschaftliche Katastrophe.

Die ersten regulären Banknoten in Europa wurden 1661 in Schweden ausgegeben, und nur zögerlich folgten mit England (1694) und Frankreich (1703) weitere Länder. Bezeichnenderweise gerieten all diese frühen Projekte innerhalb weniger Jahre aus dem Ruder. Vor allem nach dem wirtschaftlichen Desaster und den sozialen Erschütterungen, in die Frankreich 1720 durch die Papiergeld-Experimente von John Law geraten war, schwand auf lange Zeit hin in ganz Europa das Vertrauen in das neue Geld.

Ein wichtiges Kapitel in der Entwicklung des $\mathrm{Pa}$ piergeldes wurde mit dem Siebenjährigen Krieg aufgeschlagen. Infolge der enormen finanziellen Kriegsbelastungen versuchten gleich mehrere Länder, erstmalig mit der Ausgabe von Banknoten 
oder Staatspapiergeld „frisches“ Geld zu requirieren, so 1758 Russland, 1759/62 Österreich, 1766 Preußen und 1772 Sachsen. ${ }^{1}$ Im Gegensatz zu allen seinen Vorgängern beschritt Sachsen diesen neuen Weg erfolgreich. Im Folgenden soll überblicksartig diese Geschichte eigenständigen sächsischen $\mathrm{Pa}$ piergeldes und sächsischer Banknoten umrissen werden. ${ }^{2}$ Auf Ersatz- und Notzahlungsmittel, wie sie beispielsweise während der Hyperinflation im Herbst 1923 oder am Ende des Zweiten Weltkrieges auch in Sachsen zur Aufrechterhaltung des Zahlungsverkehrs notwendig waren, kann nicht eingegangen werden.

\section{Banknote oder Papiergeld?}

Eingangs ist eine kurze definitorische Abgrenzung notwendig. Während heute gemeinhin alle Geldscheine synonym als Banknoten oder Papiergeld bezeichnet werden, wurde bis ins 20. Jahrhundert hinein zwischen diesen beiden Formen unterschieden. ${ }^{3}$ Die von ihrer Entstehungszeit her älteren Banknoten wurden von speziell berechtigten Notenbanken, den sogenannten Zettel-Banken, ausgegeben. Sie waren in ihrer Anfangszeit nur Quittungen über das dafür hinterlegte Metallgeld, gegen das sie jederzeit wieder eingelöst werden konnten. Da bei einer soliden Geldpolitik erfahrungsgemäß nie alle Banknoten gleichzeitig zum Einlösen vorgelegt wurden, zog die emittierende Bank daraus Gewinn, dass sie mehr Banknoten ausgab, als sie Münzgeld eingelagert hatte. Als gesundes Maß wurde hierbei lange Zeit die Dritteldeckung angesehen, bei der ein Drittel der ausgegebenen Noten durch Edelmetallreserven gedeckt war.

Später griffen dann die Staaten selbst auf diese Form zinsloser Kredite zurück und gaben eigenes Papiergeld aus. Im Gegensatz zur Banknote war Staatspapiergeld nicht zwangsläufig gegen Münzgeld einlösbar und erhielt im Zahlungsverkehr eine gesetzliche Annahmepflicht. Im Gegenzug für diesen Zwangskurs verpflichtete sich der ausgebende Staat, sein Papiergeld bei öffentlichen Zahlungen
- insbesondere bei Steuern und Abgaben - zum Nennwert anzunehmen. Als Sicherheit diente in Erwartung zukünftiger Steuereinnahmen nur das darauf basierende Zahlungsversprechen des Staates. Jeder Untertan, der Staatspapiergeld annahm, wurde somit automatisch zum Gläubiger seines Landes. Umgekehrt besaßen die für die Staatsschulden bürgenden Landstände ein Mitbestimmungsrecht bei der Ausgabe.

Die Trennung zwischen dem von einer öffentlichen oder privaten Kasse ausgegebenen Papiergeld - daher oftmals der Name "Cassen-Schein“ oder „Cassen-Billet“ - und den von privaten oder staatlichen Banken ausgegebenen Banknoten hob sich im allgemeinen Zahlungsverkehr größtenteils auf. Hier waren der Betrag und die Sicherheit der Geldscheine bedeutsamer. Da staatliches Papiergeld sich schnell und weiträumig im Umlauf verbreiten sollte, wurde es zumeist in kleineren Nominalen zu 1, 2 oder 5 Talern ausgegeben. Bei der Zulassung von Notenbanken waren die jeweiligen Landesherren hingegen bestrebt, eine zu große Verbreitung innerhalb der Bevölkerung zu vermeiden, und genehmigten sehr oft nur die Ausgabe besonders hoher Nominale, sodass der Einsatz von Banknoten vor allem dem Großhandel und dem Bankenverkehr vorbehalten blieb.

Die Bevölkerung stand den neuen Zahlungsmitteln lange Zeit äußert skeptisch gegenüber. Zu lange waren es die Menschen einfach anhand ihrer Münzen gewohnt, dass Geld immer nur so viel wert war, wie es Silber oder Gold enthielt. Das dem Material nach wertlose Papiergeld mochte den Zahlungsverkehr oder Geldtransport noch so erleichtern, es war nichts, das als langfristiger Vermögenswert im Sparstrumpf verschwand.

\section{Frühe sächsische Notenbankprojekte und die „Brühlsche Papiergeldwirtschaft“ im Augusteischen Zeitalter}

Im Kurfürstentum Sachsen war es wie so oft der gestiegene Finanzbedarf Augusts des Starken (1670-1733), der schon früh zur Suche nach inno-

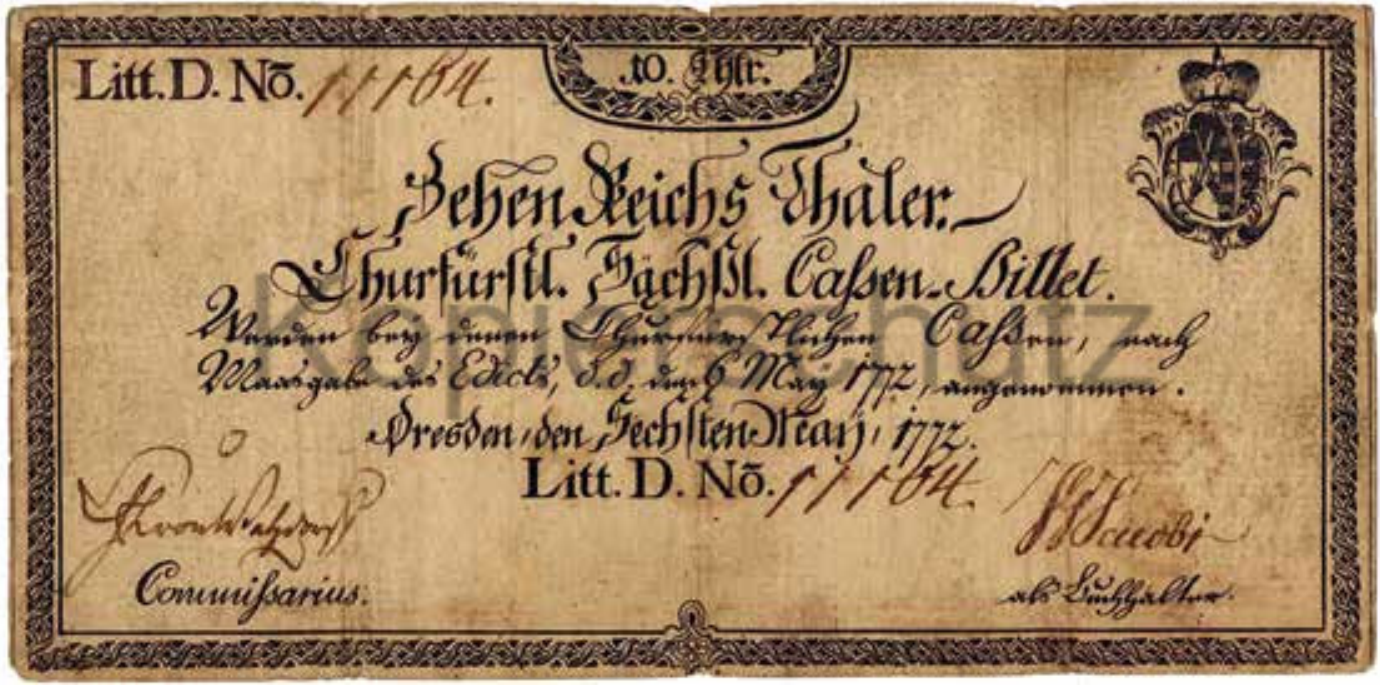

1 Einen weltweiten Überblick bieten immer noch $\mathrm{Al}$ bert Pick: Papiergeld. Braunschweig 1967; Albert Pick: Papiergeldlexikon. Regenstauf 1992.

2 Aus Platzgründen kann nur summarisch auf die wichtigste verwendete Literatur verwiesen werden: Das deutsche Staatspapiergeld. [Reprint eines Buches von 1901] Regenstauf 1993; Pick 1967 (wie Anm. 1), S. 167-174; Karl Weigel: Die Kurfürstlich und Königlich Sächsischen Kassenbilletts 1772-1874. In: Numismatische Hefte 40 (1989), S. 63-77; Heinz Fengler: Geschichte der deutschen Notenbanken vor Einführung der Mark-Währung. Regenstauf 1992; Lienhard Buck: Die „chursächsischen Cassen=Billets“. In: Der Geldscheinsammler 10 (1996), Nr. 9, S. 36-39 und 11 (1997), Nr. 2, S. 32-36; Frank Metasch: Moderne Formen staatlicher Geldschöpfung. In: Dresdner Hefte 31 (2013), Heft 114, S. 72-80; Christian Müller: Die Königlich Sächsischen Cassenbillets [1840/1855]. In: Freiberger Münzblätter 22 (2013), S. 9-20 und 23 (2014), S. 2333. Im Folgenden werden nur darüber hinausgehende Titel sowie Zitate belegt.

3 Beispielsweise Andreas Kaiser: Das Papiergeld des Kurfürstentums Hessen. Marburg 2004, S. 17-22; Arnold Keller: Das Papiergeld der Altdeutschen Staaten. Berlin 1953, S. 15 f.

Kurfürstlich Sächsisches Kassenbillett, 10 Reichstaler, 6. Mai 1872

๑ Deutsche Bundesbank Frankfurt am Main 
4 Pick 1967 (wie Anm. 1), S. $167 \mathrm{f}$.

5 Lienhard Buck: Die Münzen des Kurfürstentums Sachsen 1763 bis 1806. Berlin 1981, S. 14.

6 Vgl. den zuletzt erschienenen Sammelband: Dirk Syndram/Claudia Brink (Hrsg.): Die königliche Jagdresidenz Hubertusburg und der Frieden von 1763. Dresden 2013.

7 Walther Däbritz: Die Staatsschulden Sachsens in der Zeit von 1763 bis 1837. Leipzig 1906, S. 37, 64.

8 Mit weiteren Literaturverweisen: Winfried Müller: Das sächsische Rétablissement nach 1763. In: Dresdner Hefte 31 (2013), Heft 114, S. 14-24.

9 Horst Schlechte: Die Staatsreform in Kursachsen 17621763. Berlin 1958, S. 43. vativen Wegen der Kapitalbeschaffung führte. Hierzu zählen auch verschiedene Bestrebungen, in der Handels- und Messestadt Leipzig eine Notenbank zu errichten. Letztendlich wurde zwar keines dieser Konzepte realisiert, aber immerhin gilt die im Jahre 1700 durch den Franzosen Olivier Du Mont angeregte Gründung einer Banknoten emittierenden „National-Banc“ als das erste deutsche Notenbankprojekt überhaupt. ${ }^{4}$

Unter dem Sohn Augusts des Starken, dem Kurfürsten Friedrich August II. (1696-1763), wurde dann in weitaus stärkerem Maße auf neue Mittel zur Kreditschöpfung zurückgegriffen. In immer größerem Umfang ließ der für die Finanzpolitik zuständige Premierminister Heinrich Graf von Brühl (1700-1763) verschiedene übertragbare Staatsschuldverschreibungen ausgeben, was schon von den Zeitgenossen abwertend als „Brühlsche Papiergeldwirtschaft" betitelt wurde. ${ }^{5}$ Im Zahlungsverkehr spielten diese Schuldverschreibungen noch keine wirkliche Rolle und sind aus heutigem Verständnis allenfalls als Papiergeld-Vorläufer anzusehen. Trotzdem sind es gerade diese neuartigen, verzinslichen Wertpapiere, die die sächsische Staatsverschuldung in bislang unerreichte Höhe trieben, und die - da sie sich mehrheitlich in der Hand ausländischer, insbesondere auch preußischer Untertanen befanden - im Siebenjährigen Krieg sogar zum Gegenstand der abschließenden Friedensverhandlungen in Hubertusburg werden sollten. ${ }^{6}$

Am Ende des Siebenjährigen Krieges stand Sachsen nicht nur kurz vor dem politischen Zusammenbruch, sondern eben auch am Rande des finanziellen Ruins. Allein die Staatsschulden hatten eine Höhe von 49 Millionen Talern erreicht, und damit weitaus mehr als das Zehnfache der 1763 erwarteten Staatseinnahmen. ${ }^{7}$ Entsprechend stand im Mittelpunkt des sächsischen Wiederaufbauprogramms - des Rétablissements - die systematische Abtragung der Staatsschulden. ${ }^{8}$

\section{Die sächsischen Kassenbillets von der Einführung 1772 bis zu den Gebiets- abtretungen 1815}

Gerade die konsequente Schuldentilgung schränkte den finanziellen Spielraum Sachsens nach 1763 jedoch stark ein. Als dann 1771/72 einer Hungersnot in Sachsen rund 100.000 Menschen zum Opfer fielen und es zu einer regelrechten „Finanz- und Wirtschaftskrise“" kam, empfahl das Obersteuerkollegium dem bestehenden Kapitalmangel mit der Ausgabe von Staatspapiergeld entgegenzuwirken.

Vielen Autoren gelten die 1772 eingeführten „Churfürstlich Sächsischen Cassen-Billets“ als erstes deutsches Papiergeld überhaupt. Dass nicht den zehn Jahre älteren „Wiener-Stadt-Banco-Zetteln" dieses Prädikat gebührt, ist ein Ergebnis der auf Preußen fokussierten „kleindeutschen Lösung“ des 19. Jahrhunderts, der zufolge Österreich nicht mehr als Teil eines deutschen Nationalstaa- tes verstanden wurde. Auch bei den angesprochenen Notenbankprojekten hätte die Donaumonarchie ältere Beispiele zu bieten.

1772 barg die Einführung von Papiergeld noch viele Risiken. Die wenigen europäischen Beispiele, an denen sich Sachsen orientieren konnte, versprachen nur bedingten Erfolg. Vor allem die französischen Erfahrungen verdeutlichten, dass eine falsch in Angriff genommene Papiergeldpolitik sich schnell ins Gegenteil verkehren und die Wirtschaft auf Jahre, wenn nicht gar Jahrzehnte hin schädigen konnte. Ziel des Staates musste es daher sein, die neue Papiergeldpolitik so zu gestalten, dass die Kassenbillets sich schnell im Zahlungsverkehr durchsetzten. Denn nur, wenn das zusätzlich in Umlauf gebrachte Geld auch dort verlieb und nicht umgehend wieder eingelöst wurde, konnte das ganze Projekt Erfolg haben. Andererseits durfte aber auch nicht mehr Geld ausgegeben werden, als das Land mit seinem jeweiligen ökonomischen Entwicklungsstand aufnehmen konnte.

Als konkretes Vorbild dienten Sachsen die bereits erwähnten, zehn Jahre zuvor in Österreich ausgegebenen Banco-Zettel der Wiener Stadtbank, die sich aufgrund ihrer hohen Akzeptanz schnell in den gesamten habsburgischen Erblanden verbreitet hatten. Als Deckung für das in Sachsen im Umfang von bis zu 1,5 Millionen Reichstalern zu emittierende Papiergeld wurden die Einkünfte der staatlichen Verbrauchssteuer - der Landakzise bestimmt. Bis auf wenige Ausnahmen sollten die neuen Geldscheine ab dem 1. Oktober 1772 von allen öffentlichen Kassen „wie baares“ angenommen und ausgegeben werden. Um das Vertrauen der Bevölkerung nicht von vornherein aufs Spiel zu setzen, wurde auf einen vollständigen Zwangskurs verzichtet.

Priorität bei der Einbürgerung der Geldscheine in den Zahlungsverkehr besaß sicherlich die Bestimmung, dass zukünftig sämtliche Steuern und Abgaben an den Staat, die eine Summe von zwei Reichstalern überstiegen, zur Hälfte in Kassenbillets zu leisten waren. Nur in Ausnahmefällen durfte diese Quote unterschritten werden, sodass jeder abgabepflichtige Einwohner gezwungen war, sich mehrmals im Jahr das neue Papiergeld zu beschaffen. Um auch die letzten Bedenken zu zerschlagen, konnten die Kassenbillets in der eigens hierzu errichteten Dresdner Hauptauswechslungskasse jederzeit in Münzgeld umgetauscht werden. Eine geringe Gebühr verhinderte aber erfolgreich, dass hiervon allzu häufig Gebrauch gemacht wurde.

Die Gehälter für seine höheren Beamten und Militärs beglich der Staat nun ebenfalls zunehmend zur Hälfte mit dem neuen Papiergeld. Selbst die Mitglieder der kurfürstlichen Familie erhielten schon 1772 die Hälfte der Zahlungen für ihre Privatschatullen in Kassenbillets. Schon aus eigenem Interesse musste daher die Staatsspitze darauf bedacht sein, dass das Papiergeld nicht an Wert verlor.

Trotz aller anfänglichen Skepsis und der fehlenden Erfahrung im Umgang mit dem neuen Geld stieg das Vertrauen der Bevölkerung schnell an. Bereits 


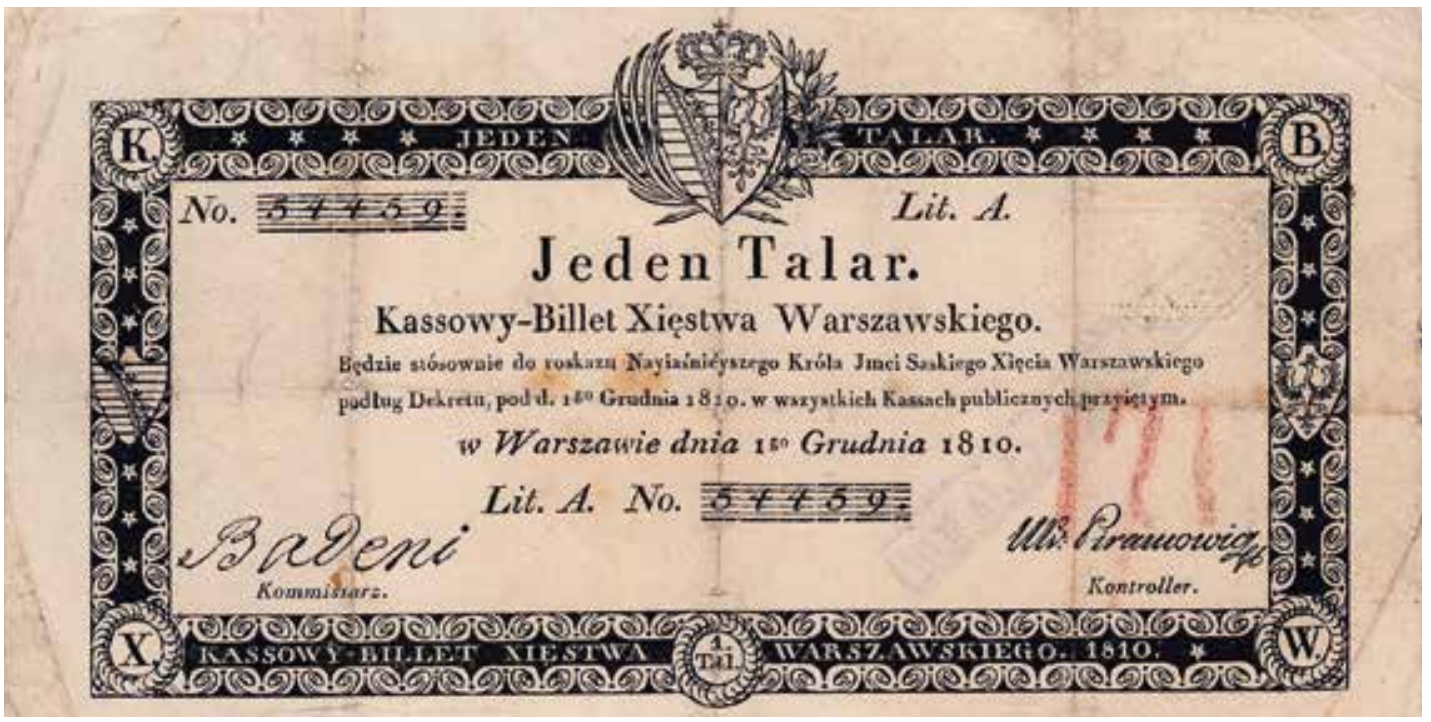

Kassenbillet aus dem Herzogtum Warschau, 1 Taler, 1. Dezember 1810 Foto: Frank Metasch

1778 besaßen die Billets einen so hohen Wechselkurs, dass ihr Umtausch in Münzen einen unnötigen finanziellen Verlust bedeutet hätte. Auch die Regierung war Ende 1778 vom Erfolg ihres Papiergeldes überzeugt und kündigte am 30. Dezember an, das Wirkungsfeld noch zu erweitern. Obwohl sich zu diesem Zeitpunkt die Gesamtsumme von 1,5 Millionen Reichstalern noch gar nicht im Umlauf befand, war die Regierung zudem der Meinung, dass mittlerweile jeder Einwohner problemlos an die Kassenbillets kommen könne. Wer trotzdem bei den öffentlichen Kassen Scheine für seine Steuerzahlungen eintauschen wollte, musste ab dem 1. April 1779 ein Aufgeld von 6 Pfennigen pro Reichstaler in Kauf nehmen.

Leider lassen sich beim derzeitigen Forschungsstand kaum Aussagen treffen, wie hoch eigentlich neben den eingesparten Zinsen der gesamtwirtschaftliche Gewinn war, den der Staat aus seinem Papiergeld zog. Insgesamt soll sich für die sächsischen Staatskassen der mit den Kassenbillets erwirtschaftete Gewinn zwischen 1772 und 1806 auf eine Million Reichstaler belaufen haben. ${ }^{10}$ Vielleicht war es deshalb auch kein „Zufall“, dass der sächsische Staatshaushalt schon zwei Jahre nach Einführung der Billets erstmals seit Jahrzehnten wieder einen Überschuss aufwies. ${ }^{11}$

Damit das Staatspapiergeld sich schnell unter der Bevölkerung verteilen konnte und auch „kleinere“ Steuerzahlungen möglich waren, lauteten 97 Prozent aller Kassenbillets auf die für den allgemeinen Umlauf wichtigen Nominale zu 1, 2 und 5 Reichstalern. Nichtsdestotrotz darf nicht vergessen werden, dass selbst eine 1-Reichstaler-Note zu dieser Zeit einen sehr hohen Wert repräsentierte, der beispielsweise dem Wochenlohn eines Bergarbeiters entsprach. Im alltäglichen Zahlungsverkehr der einfachen Bevölkerung dürfte Papiergeld somit keine größere Rolle gespielt haben. Für größere Transaktionen, beispielsweise im Messe- und Kreditgeschäft, standen Kassenbillets zu 10, 50 und 100 Reichstalern zur Verfügung. Trotz der deutlich kleineren Auflagen - von den 50-Reichstaler-Billets sind beispielsweise nur 1.000 Stück gedruckt wor- den - deckten diese aufgrund ihres hohen Nominalwertes trotzdem 20 Prozent der ausgegebenen 1,5 Millionen Reichstaler ab.

Je länger sich das Papiergeld in regem Umlauf befand, desto größer fielen die Gebrauchsspuren aus, und umso schwerer ließen sich echte von falschen Scheinen unterscheiden. Als die Hauptauswechslungskasse kaum noch hinterherkam, die beschädigten Stücke durch neue zu ersetzen, und als auch die Anzahl der Fälschungen immer weiter zunahm, wurde für 1804 die Einführung einer neuen, grafisch aufwendiger gestalteten Serie beschlossen. Das bisherige Notenkontingent von 1,5 Millionen Reichstalern wurde auch bei den neuen Kassenbillets anfänglich beibehalten. Da aber zugunsten der für den Umlauf bedeutenderen niedrigen Wertstufen auf Nominale oberhalb von 5 Reichstalern verzichtet wurde, standen trotzdem 40 Prozent mehr Geldscheine für den Publikumsverkehr zur Verfügung. Dass sich die Kassenbillets mittlerweile im Zahlungsverkehr etabliert hatten, zeigt die Herabsetzung des Zwangsaufgeldes und der Einwechselgebühr auf einen Pfennig pro Reichstaler. Zusätzlich zur Hauptauswechslungskasse in Dresden war es jetzt auch in Leipzig möglich, die Billets in Münzgeld einzuwechseln. Die Geldscheine waren nunmehr sogar so begehrt, dass selbst an den öffentlichen Kassen ein Mangel entstand und die Billets gegen entsprechende Kosten bei anderen Bankanstalten aufgekauft werden mussten.

Mit der Aufwertung Sachsens zum Königreich von Napoleons Gnaden kehrten 1806 auch im sächsischen Papiergeldwesen unruhige Zeiten ein. Der größer gewordene, nunmehr königliche Hof und die neuen militärischen Verpflichtungen ließen den Finanzbedarf des Staates deutlich steigen. Entgegen der bisherigen soliden Papiergeldpolitik wurde daher bis 1812 die Menge der Kassenbillets sukzessive auf 5 Millionen Reichstaler aufgestockt. Nur am Rande kann hier erwähnt werden, dass nach der Übertragung des Herzogtums Warschau an den sächsischen König 1807 auch dort drei Jahre später nach sächsischem Vorbild Papiergeld eingeführt wurde.
10 Das deutsche Staatspapiergeld (wie Anm. 2), S. 72.

11 Schlechte 1958 (wie Anm. 9), S. 119. 
Königlich Sächsischer InterimsKassenschein, 1 Reichstaler, 18. Dezember 1815 - Deutsche Bundesbank Frankfurt am Main

Königlich Sächsisches Kassenbillet, 2 Reichstaler,

1. Oktober 1818 Foto: Frank Metasch
Die inflationäre Geldpolitik und die zunehmende Kriegsgefahr ließen das Vertrauen der Bevölkerung in das Papiergeld immer weiter schwinden. Als sich dann 1813 der Krieg den eigenen Grenzen näherte, setzte ein Ansturm auf die Auswechslungskassen ein, die daraufhin geschlossen werden mussten. Entsprechend fiel der Kurs der Kassenbillets gegenüber dem Münzgeld auf 79 Prozent des Nennwerts, erholte sich bis 1815 aber wieder vollständig.

Interimskassenscheine als Folge

der sächsischen Gebietsabtretungen

an Preußen 1815

Eine Besonderheit innerhalb der sächsischen Geldgeschichte, die die enge Verbindung zwischen Staatspapiergeld und Staatsschulden aufzeigt, stellen die Interimskassenscheine des Jahres 1815 dar. Auf dem Wiener Kongress, der nach dem militärischen Sieg über Napoleon eine neue Nachkriegsordnung in Europa herstellen sollte, wurde Sachsen als Verlierer behandelt und musste als
Kriegsentschädigung zwei Drittel seines Territoriums und knapp die Hälfte seiner Bevölkerung an Preußen abtreten. Preußen wiederum war verpflichtet, anteilig für die ihm zugesprochenen sächsischen Gebiete die darauf liegenden Staatsschulden zu übernehmen. Dies bezog sich auch auf die Kassenbillets, die ja einen Teil der Staatsschuld bildeten. Hinsichtlich des sächsischen Papiergeldes kam Preußen seiner Verpflichtung insofern nach, als es sämtliche 1-Reichstaler-Scheine in Höhe von 1,75 Millionen Reichstalern übernahm und zu eigenem Staatspapiergeld erklärte. In Sachsen verblieben somit nur noch Geldscheine im Umfang von 3,25 Millionen Reichstalern.

Nach zweijähriger Schließzeit öffnete dann am 18. Dezember 1815 die Dresdner Hauptauswechslungskasse wieder ihre Türen. Als Ausgleich für die im Umlauf fehlenden 1-Reichstaler-Scheine wurden 400.000 „Interims-Cassen-Scheine“ gedruckt. Um den Papiergeldkurs weiter zu stabilisieren wurde noch 1816 die Gesamtsumme an Kassenbillets auf 2,5 Millionen Reichstaler reduziert. Angesichts der schwierigen Nachkriegsver-
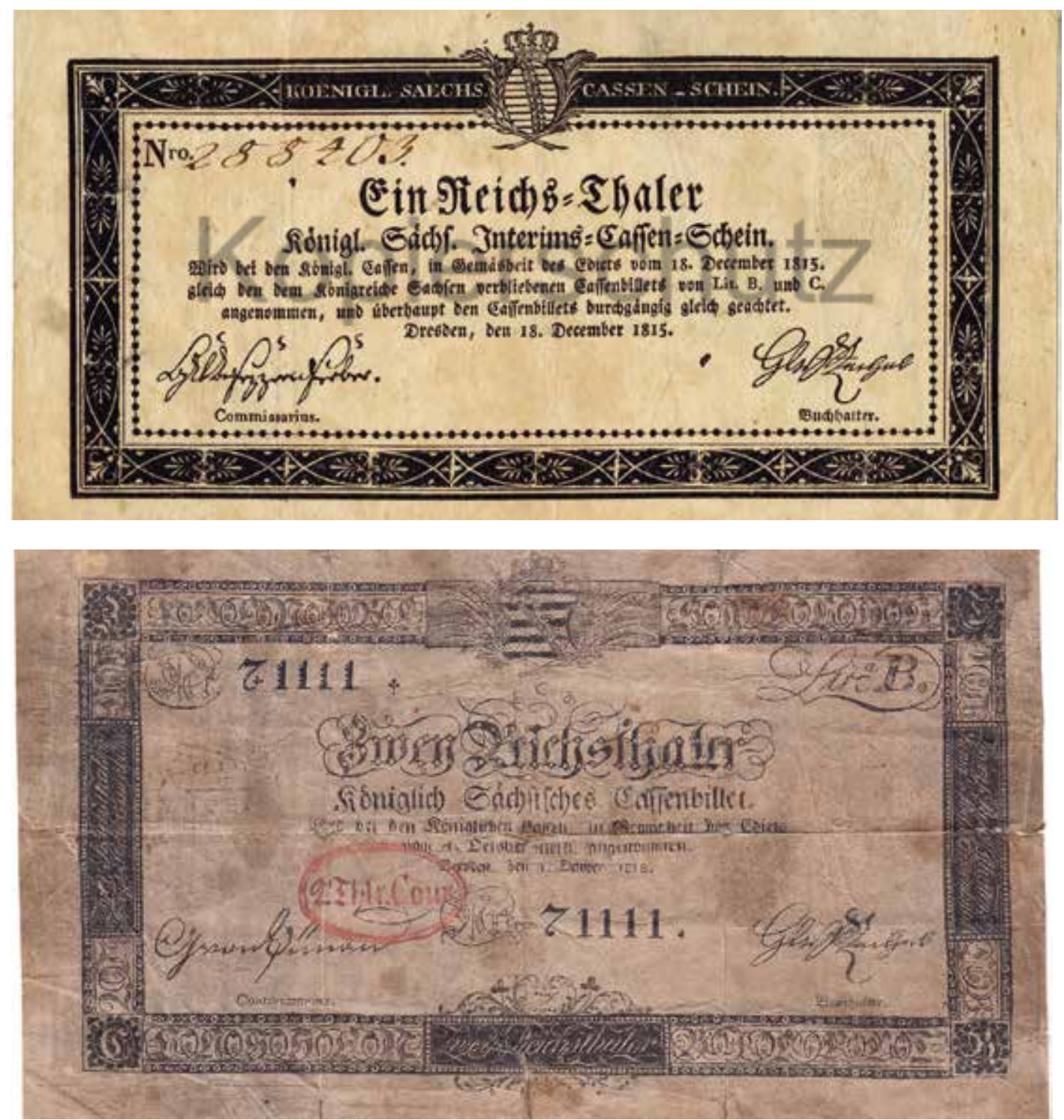
hältnisse war diese Halbierung der KassenbilletSchulden „eine wirtschafts- und währungspolitische Glanzleistung “. ${ }^{12}$

Endgültig wurde die entstandene Unordnung im Papiergeldwesen durch das Edikt vom 1. Oktober 1818 beseitigt. Die alten Scheine wurden eingezogen und im selben Gesamtbetrag von 2,5 Millionen Reichstalern durch eine neue, einheitliche Serie ersetzt. Allerdings wurde nun zugunsten höherer Auflagen bei den 1- und 2-Reichstaler-Noten auch auf das Nominal zu 5 Reichstalern verzichtet.

\section{Notenbanken und Papiergeldausgaben}

Da das Staatspapiergeld allein den Kapitalmangel auf Dauer nicht beheben konnte, rissen auch nach 1772 die Diskussionen über die Gründung von Notenbanken nicht ab. ${ }^{13}$ So schlug etwa der später geadelte Kammerrat Friedrich Wilhelm Ferber (1732-1800) 1773 die Errichtung einer staatlichen Zettelbank vor, die zu einer Banknotenemission von 2 Millionen Talern berechtigt sein sollte. Wohl sichtlich von den 1772 ausgegebenen Kassenbilletts inspiriert, sollten „gewisse Zahlungen“ zukünftig mit diesen Banknoten zu entrichten sein. In der Gründung einer Staatsbank scheint Ferber größere Handlungsspielräume als bei der Ausgabe von Staatspapiergeld gesehen zu haben. So empfahl er beispielsweise, die Höhe der Notenausgabe nicht öffentlich bekannt zu machen, um „vollkommen freie Hand zu besitzen“, und als das Projekt zu scheitern drohte, unternahm er 1774 einen erneuten Vorstoß, indem er den Kurfürsten Friedrich August III. (1750-1827) darauf hinwies, dass dieser schließlich eine solche Bank gründen („octroiren“) könne, „ohne Jemanden zu fragen“. Auch in den folgenden Jahrzehnten fanden solche Notenbankprojekte - insbesondere wenn es sich um eine staatliche Bank handeln sollte - nicht das Interesse der sächsischen Regierung, die im Gegensatz zu Preußen auch weiterhin nicht auf eine Staatsbank, sondern auf das Staatspapiergeld setzte. Eingereichte Bankprojekte wurden entweder gar nicht erst bearbeitet oder scheiterten letztlich an der notwendigen Anschubfinanzierung und den Sicherungsgarantien, die weder das Land noch die Städte übernehmen wollten.

\section{Leipziger Kassenverein 1824 und \\ Leipziger Diskonto-Kasse 1828}

1824 führte der wachsende Geldverkehr auf den Leipziger Messen dann aber doch zur erstmaligen Ausgabe von privatem Papiergeld in Sachsen. ${ }^{14}$ Mit staatlichen Vorschüssen in Höhe von $200.000 \mathrm{Ta}-$ lern versehen, gründete die Leipziger Kaufmannschaft zur Michaelismesse 1824 den Leipziger Kassenverein, der das Recht erhielt, eigene Noten ab 100 Talern aufwärts auszugeben. Von diesen Kassenscheinen in Nominalen zwischen 100 und 1.000 Talern, die durchschnittlich in einem Betrag von 629.000 Talern umliefen, sind wie von so vielen frühen Papiergeld- und Banknotenausgaben nicht einmal mehr Abbildungen geschweige denn
Originale bekannt. Um den Geschäftsverkehr des Kassenvereins zu erweiterten, wurde dessen Umwandlung in eine öffentliche, aktienbasierte Kasse angestrebt. Bereits zum 1. März 1828 ging so der Kassenverein schon wieder auf die wenige Monate zuvor als Aktiengesellschaft gegründete Leipziger Diskonto-Kasse über, die ebenfalls das Recht zur Notenausgabe ab 100 Talern besaß.

\section{Sparkassenscheine}

Angesichts des Anlasses des vorliegenden Themenheftes zum Sparkassenjubiläum soll zumindest noch ein weiteres nicht umgesetztes Geldprojekt erwähnt werden. In dem bei Sachsen verbliebenen Teil der Oberlausitz wurde 1824/25 im Zuge der Errichtung von kommunalen Sparkassen auch die Ausgabe von verzinslichen Sparkassenscheinen diskutiert. ${ }^{15}$ Die Oberlausitzer beziehungsweise sächsischen Sparkassen sollten solche Scheine je nach Vermögensverhältnis ihrer Stadt ausgeben und, da in Sachsen zu diesem Zeitpunkt nur Staatspapiergeld zu ein und zwei Talern zirkulierte, sich auf ein ergänzendes Nominal zu fünf Talern stützen. Damit aus den Scheinen aber keine Spekulationsobjekte würden, sollten sie möglichst nicht mit mehr als 2,5 Prozent verzinst werden.

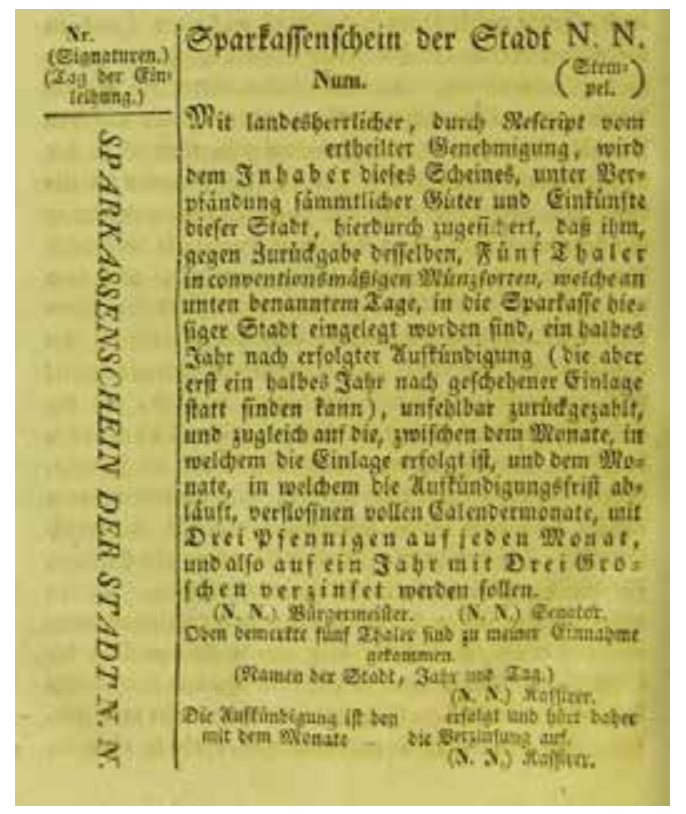

Leipzig-Dresdner Eisenbahn-Compagnie 1835

Als zentraler Handelsplatz nahm Leipzig bei Notenbanken- und Papiergeldprojekten auch weiterhin eine herausragende Rolle ein. 1835 erhielt die gegründete Leipzig-Dresdner Eisenbahn-Compagnie zur Finanzierung ihrer Bahnstrecke nicht nur das Recht, Aktien und Anleihen, sondern auch eigenes Papiergeld im Umfang von 500.000 Talern auszugeben. Zur besseren Akzeptanz im Umlauf beschränkte sich die Gesellschaft ausschließlich auf 1-Taler-Scheine. Eine erste Charge zu 100.000 Talern dieser „Leipzig-Dresdner Eisenbahn CassaScheine“ durfte die Gesellschaft mit der Eröffnung der Teilstrecke Leipzig-Wurzen am 31. Juli 1838
12 Buck 1996 (wie Anm. 2), S. 38.

13 Als Überblick, insbesondere für die nicht umgesetzten Notenbankprojekte: Heinrich von Poschinger: Die Bankentwicklung im Königreich Sachsen nach amtlichen Quellen dargestellt. In: Jahrbücher für Nationalökonomie und Statistik 26 (1876), S. 296-356 und 28 (1877), S. 73-129.

$14 \mathrm{Zu}$ den Leipziger Notenbanken vgl. auch Egon Könze: Leipziger Papiergeld und papiergeldähnliche Ausgaben. Sassenburg 2004.

15 Geißler: Ueber die Einrichtung von Sparkassen-Anstalten in Städten mittlerer Größe. In: Neues Lausitzisches Magazin 4 (1825), S. 362-371.

Textentwurf für die 1825 geplanten sächsischen Sparkassenscheine aus: Neues Lausitzisches Magazin 4 (1825), S. 368 
Erste Papiergeldausgabe der Compagnie, 1 Taler, 1838

- Deutsche Bundesbank Frankfurt am Main

Gründungsaktie der Leipziger Bank, 250 Taler, 31. August 1839 - Wikimedia

unten: Leipziger Bank, 10 Taler, 20. Juni 1864

- Deutsche Bundesbank Frankfurt am Main Leipzig-Dresdner Eisenbahn-

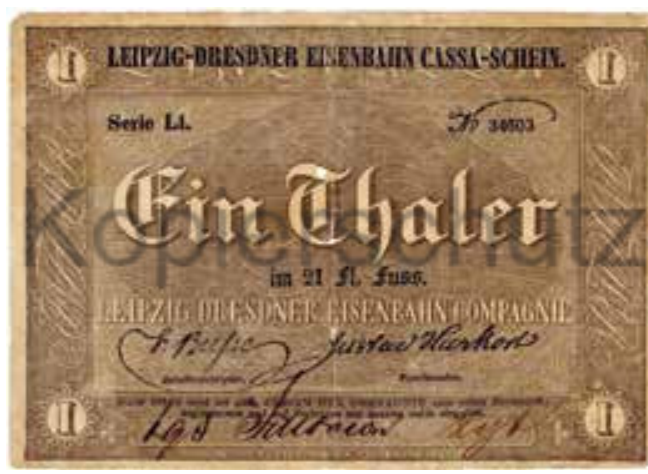

herausgeben. Die volle Summe durfte dann etappenweise mit der weiteren Vollendung der Bahnstrecken ausgegeben werden. Von diesen nun beidseitig in einer auffälligen grünen Farbe gedruckten Scheinen gab es bis 1870 mehrere Neuauflagen, um zum einen die abgenutzten Serien aus dem Umlauf zu ziehen und zum anderen im Notenbild auf Währungsveränderungen einzuge-
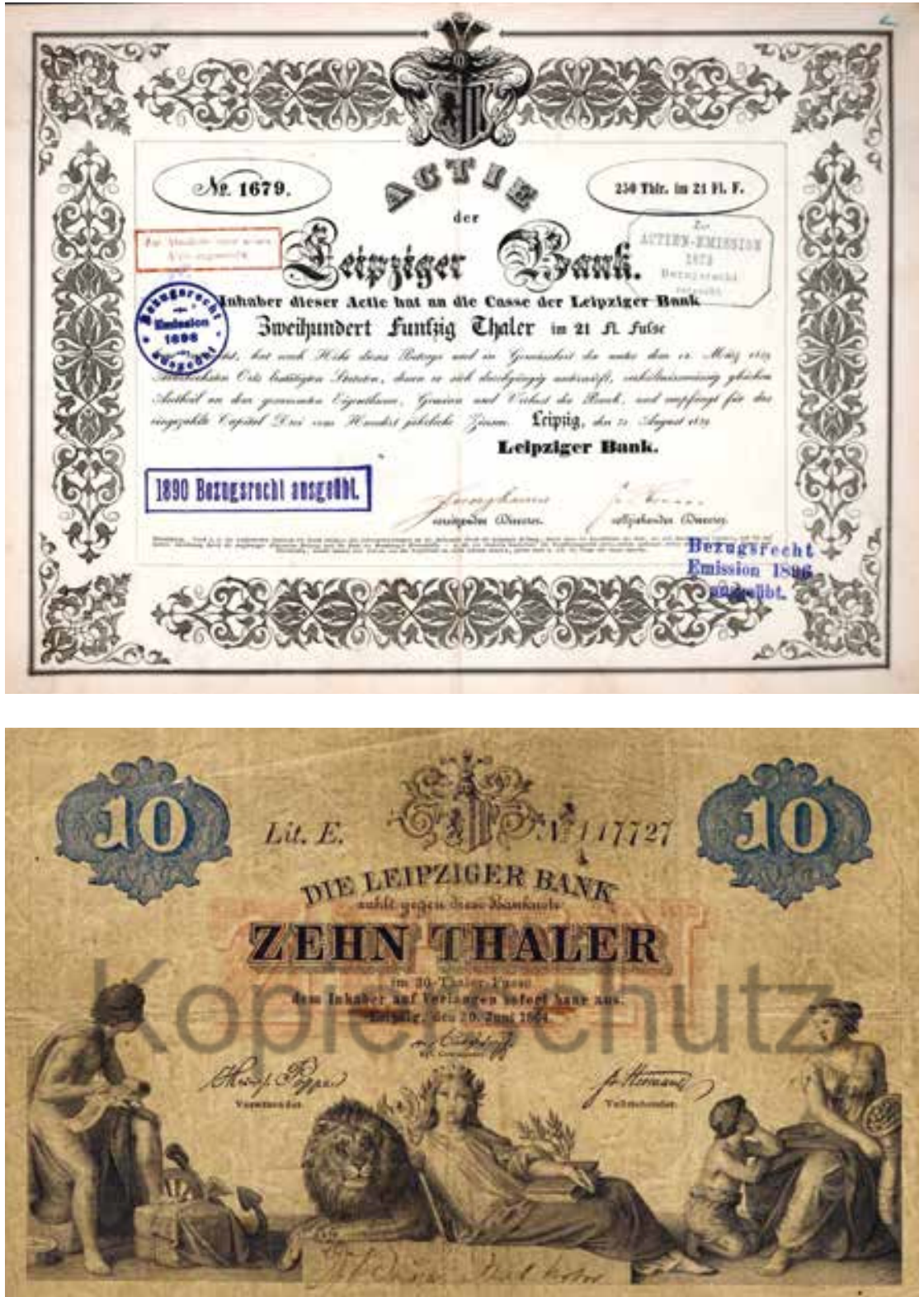

hen. An der genehmigten Gesamtsumme von 500.000 Talern sollte sich indes nichts ändern.

\section{Leipziger Bank 1838/39}

Den zunehmenden Kapitalbedarf konnten aber weder die Leipziger Diskonto-Kasse noch die Eisenbahn-Compagnie befriedigen. Die durch die Genehmigung der Diskonto-Kasse noch einmal erstarkten Pläne für eine staatliche Notenbank führten 1838/39 mit der aktienbasierten Leipziger Bank zumindest zur Gründung einer privaten Notenbank. Die Leipziger Bank übernahm plangemäß die Geschäfte und Beamten der Leipziger Diskonto-Kasse und war berechtigt, in unbegrenzter Höhe Banknoten ab einem Nennwert von $20 \mathrm{Ta}$ lern auszugeben. Als höchster Nennwert wurde 1839 sogar eine Banknote über 1.000 Taler emittiert. Erst 1863 erhielt die Bank nach mehrfachen Anläufen auch das Recht zur Ausgabe von niedrigeren 10-Taler-Scheinen.

Bis zu einem Notenumlauf von 6 Millionen Talern war der Bank eine Deckung zur Hälfte, bei Überschreitung der Summe zu zwei Dritteln vorgeschrieben. Mit dem sich schnell erweiternden Tätigkeitsfeld der Leipziger Bank reichte das anfängliche Aktienkapital von 1,5 Millionen Talern 1855 nicht mehr aus und wurde auf 3 Millionen erhöht. Kontinuierlich wuchs der Notenumlauf dann bis 1874 auf über 8 Millionen Taler an.

\section{Chemnitz-Riesaer Eisenbahn 1847}

Ähnlich wie die Leipzig-Dresdner EisenbahnCompagnie hatte 1847 auch die Chemnitz-Riesaer Eisenbahn die Genehmigung erhalten, zur Aufbringung des für den Streckenbau notwendigen Kapitals eine Anleihe über 2 Millionen Taler aufzunehmen. ${ }^{16}$ Diese mit vier Prozent verzinste Anleihe war durch ihre auf zehn Taler lautenden Obligationen wohl so handlich, dass sie in kürzester Zeit in den allgemeinen Geschäftsverkehr eindrangen. Als verschiedene Banken und Firmen öffentlich verbreiteten, die Anleihescheine beziehungsweise deren jährliche Zinscoupons wie Bargeld zu behandeln, fühlte sich im September 1847 die sächsische Regierung zum Einschreiten gefordert. Damit nicht der Eindruck entstände, es handle sich bei diesen reinen Schuldobligationen um Banknoten, wurde verlangt, deren Bareinlösung einzustellen.

\section{Landwirtschaftliche Kreditkrise und die Revolution von 1848/49}

Dass Ende der 1840er Jahre selbst reine Schuldobligationen von Banken schon wie Bargeld behandelt wurden, verweist nochmals auf den zu dieser Zeit enorm gestiegenen Finanzbedarf, der sich deutschlandweit zu einer wahren Kreditkrise entwickelt hatte. Stand schon für die wirtschaftlich führenden Städte nicht genügend Geld zur Verfügung, so waren die Auswirkungen im landwirtschaftlichen Bereich, für den sich weder die allmählich etablierenden Sparkassen noch die großen 
Banken zuständig fühlten, umso erheblicher. Mehrfach kam es daher zum Gründungsversuch von landwirtschaftlichen Kreditvereinen. Im Revolutionsjahr 1848 erhöhten die steigenden Militärausgaben und die einsetzenden Steuerausfälle auch auf staatlicher Seite den Handlungsdruck, sodass es in ganz Deutschland zu einer Vielzahl neuer Banknoten- und Papiergeldausgaben kam.

\section{Chemnitzer Stadtbank 1848}

Die Gründung der Chemnitzer Stadtbank 1848 als einer städtischen Notenbank war ein Zugeständnis der sächsischen Regierung an die Chemnitzer Forderungen während der Revolutionszeit. Da die Leipziger Bank trotz vorheriger Verpflichtung keine Zweigstelle in Chemnitz, dem industriellen Zentrum Sachsens, eröffnete, sollte die Chemnitzer Stadtbank helfen, die auf die weitere wirtschaftliche Entwicklung hemmend wirkende Kapitalnot zu lindern. Mit einem Aktienkapital von 100.000 Talern ausgestattet, stieg der Notenumlauf bis 1861 auf knapp 900.000 Taler, für die die Stadt die Sicherheiten übernommen hatte. Im Gegensatz zu vielen anderen Banken emittierte die Chemnitzer Stadtbank nur 1-Taler-Noten, was auf eine angestrebte schnelle Einstreuung in den Geldumlauf schließen lässt. Hinzu kommt als weitere Besonderheit die fehlende Ausrichtung auf eine alleinige Gewinnerzielung. Im Vordergrund stand die Förderung von Handel, Industrie und Gewerbe in Chemnitz und Umgebung.

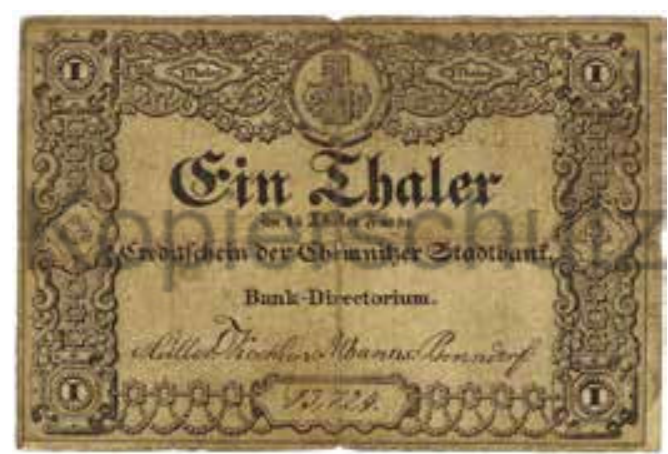

\section{Landständische Bank 1850}

Ebenfalls auf einen regionalen Wirkungskreis zielte die „Landständische Hypothekenbank des Königlich Sächsischen Markgraftums Oberlausitz zu Bautzen“, die 1844 ursprünglich nicht als Notenbank, sondern als landwirtschaftliches Kreditinstitut mit einem reinen Hypothekengeschäft gegründet worden war. Die Garantie für die Bank hatten die Oberlausitzer Stände übernommen, als Kapital wurde ihr das gesamte damalige Vermögen des Bautzener Kreises in Höhe von 550.000 Talern gegen eine jährliche Verzinsung von 3,5 Prozent zur Verfügung gestellt. Die erwirtschafteten Gewinne sollten „zum Besten“ der Oberlausitz verwendet werden.

Recht schnell erwiesen sich die ursprünglichen Statuten als unzureichend, um dem eigenen Auftrag zur Vergabe von Darlehen nachzukommen. Unter anderem fehlte dafür einfach das notwendige Bargeld. Bei der daraufhin erfolgten Umstruktu-

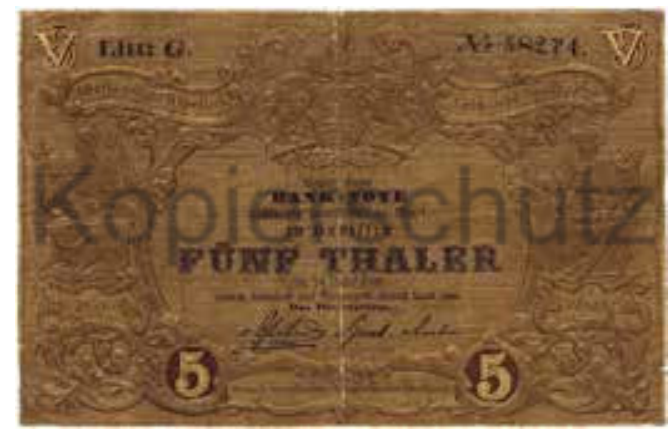

Oberlausitzer Hypothek-, Leih- und Sparbank, 5 Taler, 1850

() Deutsche Bundesbank

Frankfurt am Main

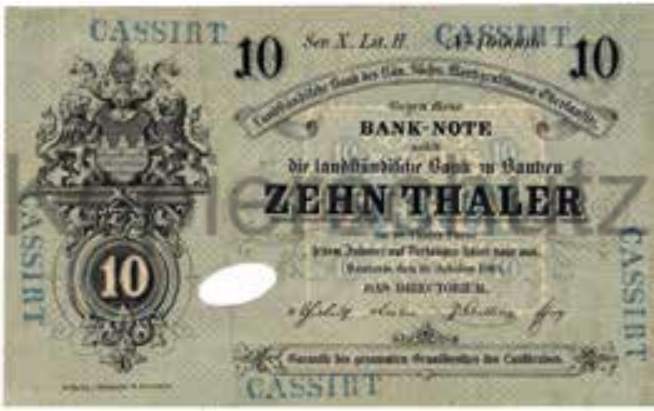

Landständische Bank des Königlich Sächsischen Markgraftums Oberlausitz, 10 Taler, 10. Oktober 1868 ( ) Deutsche Bundesbank Frankfurt am Main

rierung und Geschäftserweiterung erhielt die Bank - beziehungsweise als eine Besonderheit im Bankenwesen die für die Bank bürgenden Landstände - 1850 auch das Notenprivileg. Mit ihren neuen Banknoten konnte die Bank wohl unter anderem auch Teile des ihr von den Landständen in Form von Pfandbriefen zur Verfügung gestellten Kapitals zurückzahlen und sich so zukünftig diese Zinskosten ersparen. ${ }^{17}$ Die ersten Empfänger der Banknoten waren jedenfalls die Landstände selbst. Die Geschäfte der Landständischen beziehungsweise - wie sie im Geschäftsverkehr auch genannt wurde - Bautzener Bank entwickelten sich so erfolgreich, dass in den Folgejahren immer wieder Statutenänderungen in Hinsicht auf eine Ausdehnung des Notenkontingents erforderlich waren. Die Notenausgabe erhöhte sich so von $150.000 \mathrm{Ta}$ lern 1851 auf eine Million Taler 1871.

Zugutekommen sollte dieses Geld in Form von Grundkrediten vor allem der Land- und Forstwirtschaft. Hier profitierten also die zu den Landständen gehörenden Rittergutsbesitzer selbst mit am stärksten. Umgekehrt hatte aber gerade der Oberlausitzer Adel große Teile seines Kapitals in der Bank investiert, sodass es nicht verwunderlich ist, dass die von den Landständen getragene und kontrollierte Bank bis zu ihrer Auflösung 1945 „einen Kristallisationskern für das Sonderbewusstsein des Adels in der sächsischen Oberlausitz “18 bildete.

\section{Das Staatspapiergeld}

Am sächsischen Staat ging der angesprochene Bargeldmangel ebenfalls nicht spurlos vorüber. Eine Zäsur innerhalb der sächsischen Papiergeldpolitik brachte das Jahr 1834 mit der Aufhebung der bisherigen paritätischen Zahlung mit Kassenbillets bei allen Aus- und Einzahlungen der Staatskasse. Sofern nicht vertraglich eine Entrichtung in „klingender Münze“ zwingend war, konnte nun in be-
Chemnitzer Stadtbank,

1 Taler, 1848

(c) Deutsche Bundesbank

Frankfurt am Main
16 Vgl. Manfred Müller/Anton Geiger: Das Papiergeld der deutschen Eisenbahnen und der Reichspost. Frankenthal o. J.

17 Sofern ich hier Fengler 1992 (wie Anm. 2), S. 59 richtig verstanden habe.

18 Josef Matzerath: Adelsprobe an der Moderne. Sächsischer Adel 1763-1866. Stuttgart 2006, S. 73. 
19 Gunda Ulbricht: Finanzgeschichte Sachsens im Übergang zum konstitutionellen Staat (1763 bis 1843). St. Katharinen 2001, S. 11.

20 Auf den Berechnungen von Bernd Sprenger beruhend: Fengler 1992 (wie Anm. 2), S. 123.

Königlich Sächsisches Kassenbillet, 10 Taler, 16. April 1840

(- Deutsche Bundesbank Frankfurt am Main

Königlich Sächsisches Kassenbillet, 10 Taler, 6. September 1855 (c) Deutsche Bundesbank Frankfurt am Main liebiger Höhe mit den Kassenbillets gezahlt werden. Zudem wurde ab jetzt auch keine Gebühr mehr beim Umtausch gegen Münzgeld erhoben.

Im selben Jahr sollte mit der Gründung des Deutschen Zollvereins, dem auch Sachsen beigetreten war, die immer noch nicht erreichte monetäre Einheit Deutschlands ein gutes Stück vorangebracht werden. Der zu diesem Zeitpunkt nicht mehr nur im monetären Bereich kaum noch zu übersehenden Dominanz Preußens entsprechend, bestimmte auch die Dresdner Münzkonvention von 1838 die preußische Währung (den 14-Taler-Fuß) zur Grundlage des Zollvereinsgeldes. Entsprechend war auch auf dem sächsischen Staatspapiergeld der bisherige Reichstaler obsolet geworden und wurde zukünftig durch den Taler ersetzt. Für Sachsen bedeutete die mit dem Zollverein einhergehende Übernahme des Dezimalsystems unter anderem, dass der Taler nicht mehr wie bisher in 24 Groschen á 12 Pfennige, sondern jetzt in 30 Neugroschen á 10 Pfennige unterteilt war. Im Gegensatz zu den ab 1838 geprägten Vereinsmünzen verzögerte sich erst einmal die Ausgabe einer neuen Papiergeldserie. Die umlaufenden, bereits sehr stark abgenutzten Kassenbillets von 1818 mussten daher weiterhin ihren Dienst versehen. Sie wurden jedoch 1838 dem neuen Hartgeld gleichgestellt und als äußeres Zeichen mit einem roten Stempel versehen sowie der ganze Schein - sofern es die Festigkeit des Papieres noch zuließ - einer Blaufärbung unterzogen (vgl. das bereits abgebildete Kassenbillet von 1818 über zwei Reichstaler). Bei nicht gestempelten Scheinen musste zukünftig im Zahlungsverkehr ein Aufschlag (Aufgeld) von nicht ganz drei Prozent entrichtet werden, um die Wertdifferenz zum neuen Taler auszugleichen.

1842 wurde dann, gemäß dem Gesetz vom 16. April 1840, die alte, abgenutzte Emission von 1818 durch neue Kassenbillets ersetzt, diesmal auch wieder mit höheren Nominalen zu 5 und 10 Talern. Statt der eingezogenen 2,5 Millionen wurden direkt drei Millionen Taler in Umlauf gesetzt. Diese Zahl sollte sich
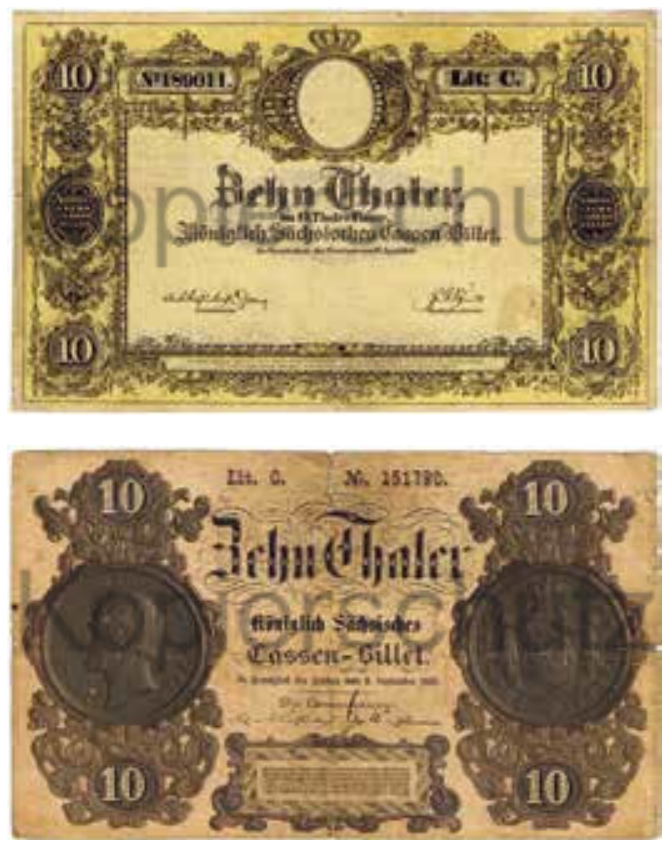

innerhalb von nur vier Jahren sogar noch auf sieben Millionen erhöhen, womit zur Jahrhundertmitte die staatliche Papiergeldemission rein rechnerisch die Höhe des jährlichen Staatshaushaltes erreicht hatte. ${ }^{19}$ Dass Sachsen damit aber im Rahmen des ökonomisch Vertretbaren geblieben war, verdeutlicht die weitere Stabilität der Kassenbillets. Das sächsische Staatspapiergeld wurde nicht nur weiterhin neben dem quantitativ deutlich bedeutenderen preuBischen Geld in weiten Teilen Deutschlands akzeptiert, es diente anderen Banken sogar als Deckung für selbst ausgegebene Banknoten, so etwa der Chemnitzer Stadtbank.

Eine solche Akzeptanz fremder beziehungsweise ausländischer Noten war in Deutschland keinesfalls selbstverständlich - ganz im Gegenteil. Viele deutsche Staaten litten damals enorm unter der Überflutung mit fremden Geldscheinen. Diese „wilden Noten“ waren oftmals nur schwer einlösbar, da sie hierfür an die ausgebende Kasse beziehungsweise Bank verschickt werden mussten. Und selbst dann war nicht gesagt, dass sie dort auch wirklich umgetauscht wurden. Viele Regierungen verboten daher - insbesondere auf einen preußischen Vorstoß hin - in den 1850er Jahren alle ausländischen Banknoten, die auf einen Nennwert von unter zehn Talern lauteten. Sachsen milderte für sein Territorium 1857 dieses Verbot dahingehend ab, dass auch alle niedrigeren Noten gültig blieben, sofern die ausgebende Bank oder Kasse eine Zweigstelle in Leipzig eröffnete und dort die Einlösung innerhalb von drei Tagen garantierte.

Die Zehn-Taler-Regel zeichnete sich vielleicht auch mitverantwortlich, dass die 1857 neu ausgegebene Kassenbillet-Serie wieder Nominale bis zu 50 Taler besaß. Andererseits zeigen diese hohen Nennwerte deutlich, dass das sächsische Staatspapiergeld nicht nur Aufgaben im einfachen Umlauf und Geschäftsverkehr erfüllen sollte. Notwendig geworden war die neue Ausgabe aber vor allem, weil das 1842 verwendete dünne Papier bereits so zerschlissen war, dass die Einlösung der Scheine beziehungsweise deren weiterer Umlauf große Probleme bereitete. Auch in puncto Fälschungssicherheit befanden sich die Kassenbillets von 1842 nicht mehr auf der Höhe der Zeit.

1857 wurden erst einmal nur die umlaufenden sieben Millionen Taler ausgetauscht. Aus dem direkt mitgedruckten Reservebestand hätten weitere eine Million Taler, gegen die entsprechende Hinterlegung von verzinslichen sächsischen Staatspapieren, in Umlauf gebracht werden können. 1861/62 wurden nochmals drei Millionen Taler gedruckt, die aber ebenfalls nur als Reserve zum Austausch verschlissener Scheine beziehungsweise der Möglichkeit, kurzfristig auf einen höheren Bedarf reagieren zu können, dienten. Notwendig wurde dies 1866 im sogenannten Deutschen Krieg gegen Österreich, als Sachsen auf seine gesamte noch vorhandene Kassenbillet-Reserve in Höhe von rund 2,8 Millionen Talern zurückgreifen musste.

1857 trat mit dem Wiener Münzvertrages nun unter anderem auch Österreich der Münzvereinigung im Rahmen des Deutschen Zollvereins bei. Der nun eingeführte 30-Taler-Fuß hatte aufgrund der nahezu 


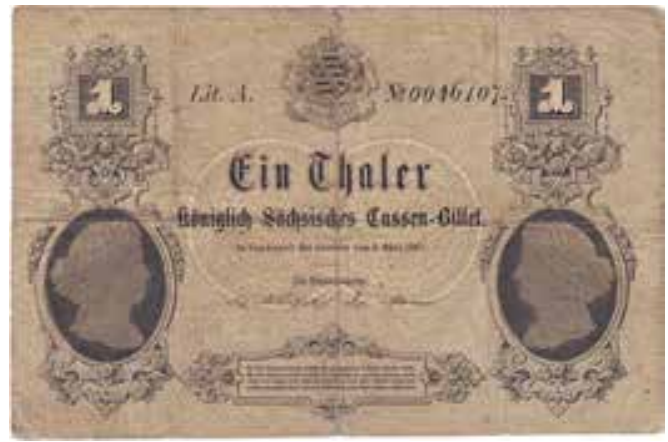

identischen Umrechnung zum bisherigen 14-TalerFuß auf die sächsischen Kassenbillets keine Auswirkungen. Allerdings verzichtet man jetzt beim Staatspapiergeld gleich auf die Angabe eines Münzfußes. So dann auch 1867 bei der letzten Ausgabe sächsischer Kassenbillets, von denen direkt zwölf Millionen Taler in Nominalen zu 1, 5 und 10 Talern in Umlauf gegeben wurden. Weitere sechs Millionen dienten als „Reservequantum“.

\section{Leipziger Kassenverein 1867 und Sächsische Bank zu Dresden 1865}

Im selben Jahr der letzten Ausgabe neuer Kassenbillets 1867 kam es zur neuerlichen Gründung eines Leipziger Kassenvereins, der im Umfang von einer Million Taler 100-Taler-Noten emittierte. Weitaus bedeutsamer war jedoch die zwei Jahre zuvor erfolgte Gründung der Sächsischen Bank zu Dresden. Diese hatte zunächst für die Dauer von 25 Jahren ein unbegrenztes Notenprivileg erhalten, musste aber im Gegenzug nicht nur die übliche Ein-DrittelDeckung in Gold oder Silber, sondern auch die restlichen zwei Drittel durch Wechsel garantieren.

Das Startkapital der Sächsischen Bank bestand aus fünf Millionen Talern, die durch die Ausgabe von Aktien erbracht wurden. Der Ansturm auf die Bankaktien war dabei, ähnlich wie bei vielen Eisenbahngesellschaften, so groß, dass diese hundertfach überzeichnet waren. Da die Sächsische Bank nicht nur eine regionale Ausrichtung auf Dresden, sondern auf das gesamte Land besaß und entsprechend in den Folgejahren ein größeres Filialnetz errichtete, stieg das Notenkontingent kontinuierlich an. Aus Banknoten im Gegenwert von 2,6 Millionen Talern waren 1874 bereits über 31 Millionen Taler geworden - also mehr, als an allen anderen sächsischen Banknoten und dem Staatspapiergeld zusammen umlief.

\section{Reichsgründung 1871}

Der industriellen Revolution mit ihrem riesigen Kapitalbedarf verdankte letztlich auch das Papiergeld seinen Siegeszug. Hatten beim Einsetzen der industriellen Revolution in Deutschland die Geldscheine gerade einmal zehn Prozent des Umlaufs von Münzen erreicht, lag diese Quote 1849 schon bei knapp 20 und 1857 bei 35 Prozent. Beim Übergang in die Phase der Hochindustrialisierung mit der Gründung des deutschen Kaiserreiches 1871 lief dann erstmalig mehr Papier- als Metallgeld um. ${ }^{20}$
Die Reichsgründung führte zu einer grundlegenden Neuordnung des Geldwesens. Als Bezeichnung für die neue Währung hatte man sich - auf einen früheren sächsischen Vorschlag von 1838 hin - auf die neutrale Bezeichnung „Mark“ verständigt, um weder die norddeutschen Taler- noch die süddeutschen Guldenländer zu übervorteilen. Für die auch
Königlich Sächsisches

Kassenbillet, 1 Taler, 2. März 1867

Foto: Frank Metasch
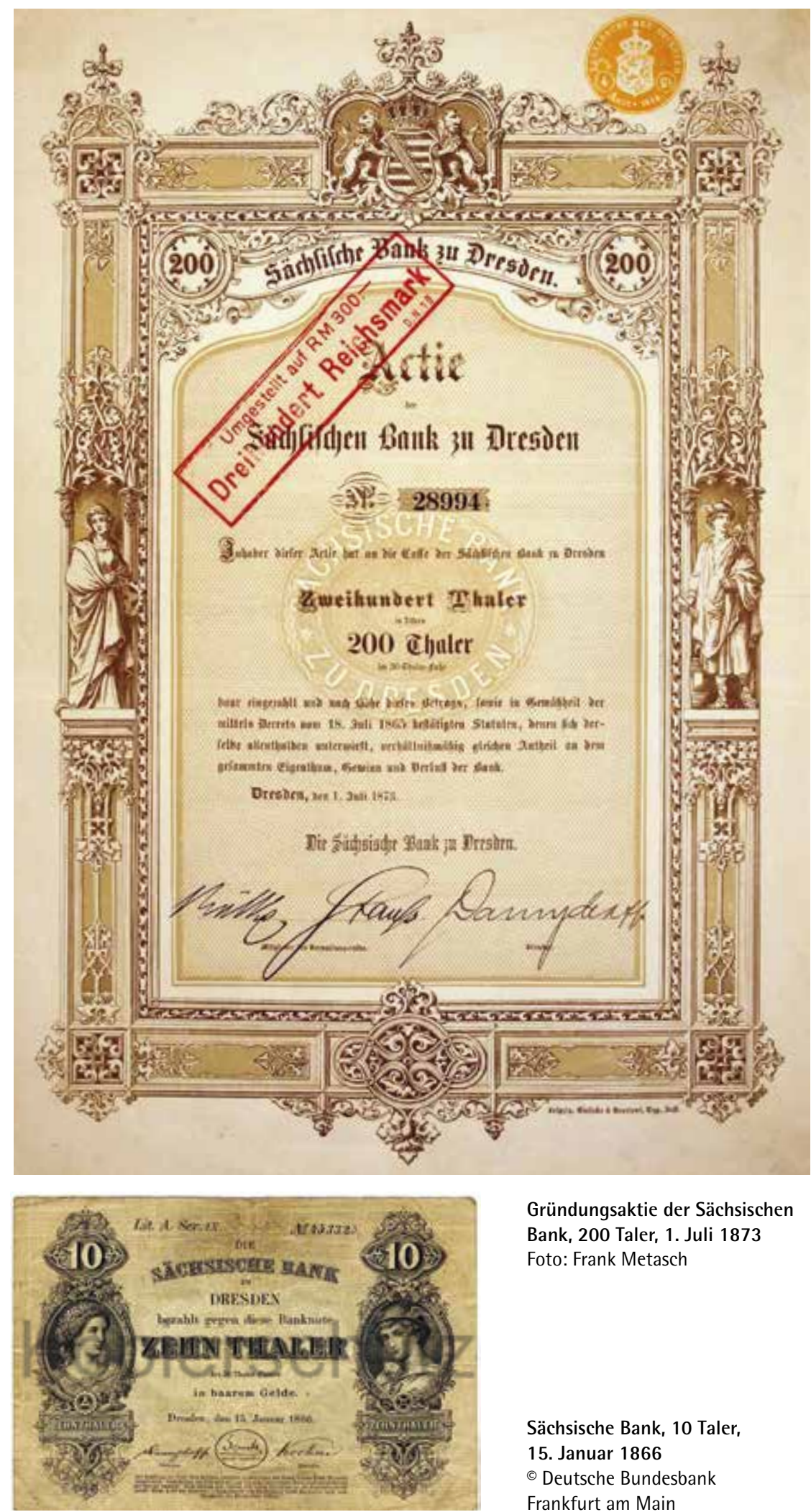

Gründungsaktie der Sächsischen Bank, 200 Taler, 1. Juli 1873 Foto: Frank Metasch

Sächsische Bank, 10 Taler, 15. Januar 1866

- Deutsche Bundesbank Frankfurt am Main 
21 Z. B. Niklot Klüßendorf: Kleine Münz- und Geldgeschichte von Hessen in Mittelalter und Neuzeit. Marburg 2012, S. 148-154; Bernd Sprenger: Das Geld der Deutschen. 3. Auflage Paderborn u.a. 2002, S. 181-186.

links: Reichskassenschein, 20 Mark, 11. Juli 1874 ๑ Deutsche Bundesbank Frankfurt am Main

rechts: Leipziger Kassenverein, 500 Mark, 31. März 1875, Probedruck der Vorderseite ๑ Deutsche Bundesbank, Frankfurt am Main

22 Mit farbigen Abbildungen dieser seltenen sächsischen Noten: Hans-Ludwig Grabowski: Die deutschen Banknoten ab 1871. 21. Auflage Regenstauf 2018.

Sächsische Bank, 10 Billionen Mark, 15. November 1923 Foto: Frank Metasch

Sächsische Bank, 50 Reichsmark, 11. Oktober 1924 Foto: Frank Metasch

Autor

Dr. Frank Metasch Institut für Sächsische Geschichte und Volkskunde

Zellescher Weg 17, 01069 Dresden in Sachsen gültige Talerwährung bedeutete das die recht einfache Umrechnung, dass ein bisheriger Taler drei Mark entsprach.

Für die papiernen Zahlungsmittel neue, reichseinheitliche Lösungen zu finden, gestaltete sich deutlich schwieriger als beim Münzgeld, da die einzelnen Staaten auf diese Möglichkeit, sich selbst zinsfreie Kredite zu verschaffen, nicht verzichten wollten. ${ }^{21}$ Das bisherige Staatspapiergeld wurde per Gesetz vom 30. April 1874 durch eine einheitliche

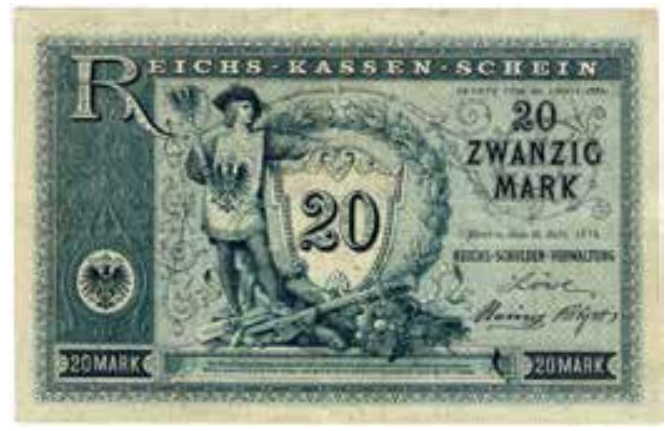

neue Ausgabe von Reichspapiergeld in Höhe von 120 Millionen Mark ersetzt: den Reichskassenscheinen. Das Reich übernahm damit die bisherigen Schulden der einzelnen Länder als Teil der Reichsschuld und verteilte die neuen Reichskassenscheine zu 5, 20 und 50 Mark nach einem an der Bevölkerungszahl orientierten Schlüssel auf die einzelnen Länder. Mit diesem Schritt endete auch in Sachsen die Epoche eigenen Staatspapiergeldes.

Den weiteren Umlauf der Banknoten regelte das Bankgesetz vom 14. März 1875. Das reichsweite Notenkontingent wurde auf 385 Millionen Mark festgesetzt, wovon rund 65 Prozent auf die aus der Preußischen Bank hervorgegangene neue Reichsbank entfielen. Sollte zukünftig eine der bisherigen Notenbanken auf ihr Notenrecht verzichten beziehungsweise sollte dieses fristgemäß erlöschen, fiel deren Kontingent automatisch an die Reichsbank. Wer ein höheres Kontingent als vorgesehen in Anspruch nehmen wollte, musste darauf eine spezielle Steuer entrichten. Die neuen Banknoten, die auf Beträge von mindestens 100 Mark lauten mussten, liefen nun aber gleichberechtigt mit den Reichsbanknoten um und waren nicht mehr auf das eigene Land beschränkt.

Erreichten die Emissionen der deutschen Privatbzw. Ländernotenbanken zwischen 1872 und 1874 mit über 400 Millionen Mark sogar noch einen Rekordwert, so sollte sich diese Summe allein in den nächsten zwei Jahren auf drastische Weise halbieren. Die hohen Deckungsvorschriften führten dazu, dass viele Banken ihr Notenrecht nicht mehr wahrnahmen. In Sachsen verzichtete 1875 die Leipziger Bank direkt auf ihr Notenprivileg. Die anderen sächsischen Banken stellten ihre Ausgaben auf die geforderten Nennwerte zu mindestens 100 Mark um, gaben dann aber in den Folgejahren ebenfalls auf: 1878 die Leipzig-Dresdner Eisenbahn-Compagnie, 1890 der Leipziger Kassenverein, 1891 die Chemnitzer Stadtbank und 1906 die Landständische Bank. ${ }^{22}$
Die Sächsische Bank gehörte damit ab 1906 - neben der Badischen Bank sowie der Bayerischen und der Württembergischen Notenbank - zu den letzten vier Länderbanken mit eigenen Banknoten. Auch sie geriet so in die Wirren der Inflationszeit und musste ab Juli 1922 in immer schnellerer Folge neue, höhere Banknotenwerte emittieren, zuletzt im November 1923 als höchstes Nominal eine Banknote über 10 Billionen Mark - einer 1 mit elf Nullen.

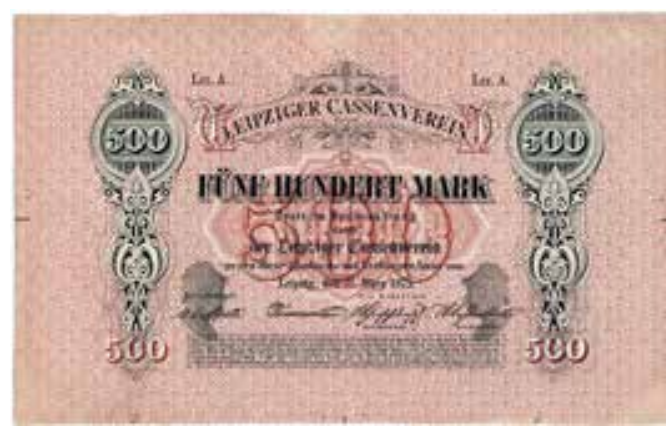

Nach der Währungsstabilisierung und der Einführung der Reichsmark gab auch die Sächsische Bank im Herbst 1924 zwei neue Banknoten zu 50 und 100 Reichsmark aus. Mit den gewählten Motiven des Malers der Romantik Ludwig Richter (18031884) und des Aufklärers Gotthold Ephraim Lessing (1729-1781) wurde hierbei auf zwei populäre sächsische Identifikationsfiguren zurückgegriffen.
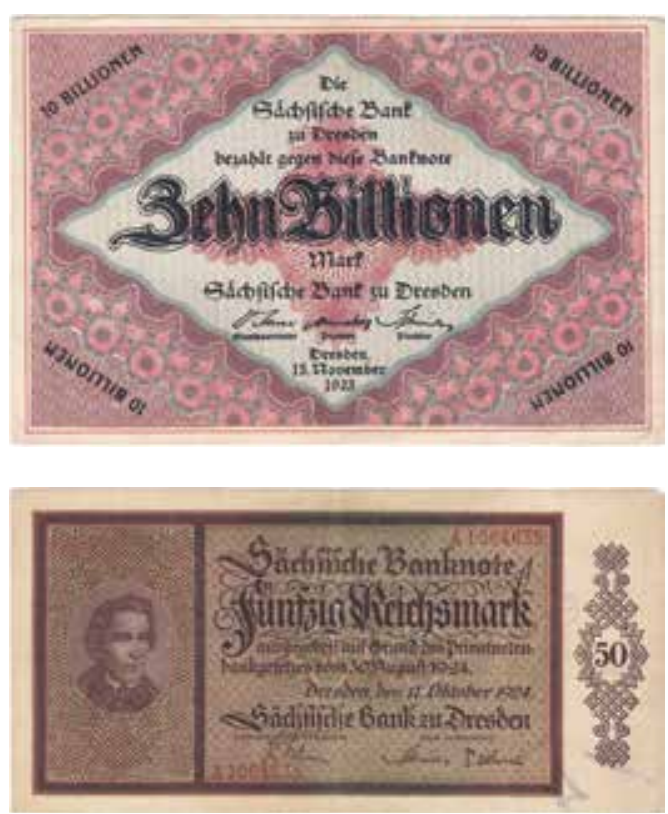

Wie auch den anderen drei Landesbanken wurde der Sächsischen Bank 1935 durch das Reichswirtschaftsministerium das Notenrecht entzogen. Mit der Außerkurssetzung ihrer Banknoten am 2. April 1936 endete in Sachsen die über 160-jährige Geschichte eigener Notenausgaben - einer Geschichte, ohne die wohl nicht nur die wirtschaftliche Entwicklung des Landes anders verlaufen wäre. 